\title{
On the Convergence of Monotone Lattice Matrices*
}

\author{
Jing Jiang ${ }^{1}$, Lan Shu ${ }^{2}$, Xin'an Tian ${ }^{1}$ \\ ${ }^{1}$ School of Mathematical Sciences, University of Electronic Science and Technology of China, Chengdu, Sichuan, China \\ ${ }^{2}$ The College of Mathematics and Statistics, Chongqing University of Arts and Sciences, Chongqing, China \\ Email: jiangjingstu@163.com
}

Received May 29, 2013; revised June 3, 2013; accepted June 4, 2013

Copyright (c) 2013 Jing Jiang et al. This is an open access article distributed under the Creative Commons Attribution License, which permits unrestricted use, distribution, and reproduction in any medium, provided the original work is properly cited.

\begin{abstract}
Since lattice matrices are useful tools in various domains like automata theory, design of switching circuits, logic of binary relations, medical diagnosis, markov chains, computer network, traffic control and so on, the study of the properties of lattice matrices is valuable. A lattice matrix $A$ is called monotone if $A$ is transitive or $A$ is monotone increasing. In this paper, the convergence of monotone matrices is studied. The results obtained here develop the corresponding ones on lattice matrices shown in the references.
\end{abstract}

Keywords: Distributive Lattice; Lattice Matrix; Convergence

\section{Introduction}

In the field of applications, lattice matrices play major role in various areas such as automata theory, design of switching circuits, logic of binary relations, medical diagnosis, markov chains, computer network, traffic control (see e.g. [1]). Since several classical lattice matrices, for example transitive matrix, monotone increasing matrix, nilpotent matrix, have special applications, many authors have studied these types of matrices. In fact, a transitive matrix can be used in clustering, information retrieval, preference, and so on (see e.g. [2,3]); a nilpotent matrix represents an acyclic graph that is used to represent consistent systems and is important in the representation of precedence relations (see e.g. [4]). Recently, the transitive closure of lattice matrix has been used to analyze the maximum road of network. In this paper, we continue to study transitive lattice matrices and monotone increasing matrices. The main results obtained in this paper develop the previous results on transitive lattice matrices [5] and monotone increasing matrices [6].

\section{Definitions and Preliminaries}

At this section, we shall give some definitions and lemmas. Let $(P, \leq)$ be a partially ordered set (simply denoted by poset) and $x, y \in P$. If $x \leq y$ or $y \leq x$ then $x$

\footnotetext{
*This work was supported by the Foundation of National Nature Science of China (Grant No.11071178) and the Fundamental Research Funds for the Central Universities.
}

and $y$ are called comparable. Otherwise, $x$ and $y$ are called incomparable, noted by $x \| y$. If for any $x, y \in P, x$ and $y$ are comparable, then $P$ is called a chain. An unordered poset is a poset in which $x \| y$ for all $x \neq y$. A chain $c$ in a poset $P$ is a nonempty subset of $P$, which, as a subposet, is a chain. An antichain $C$ in a poset $P$ is a nonempty subset which, as a subposet, is unordered. A lattice is a poset in which every two elements have a unique least upper bound and a unique greatest lower bound. For any $x$ and $y$ in $L$, the least upper bound and the greatest lower bound will be denoted by $x \vee y$ and $x \wedge y$, respectively. It is clear that any chain is a lattice, which is called a linear lattice. It is obvious that if $(L, \leq)$ is a linear lattice (especially, the fuzzy algebra $[0,1]$ or the binary Boolean algebra $\left.B_{1}=\{0,1\}\right)$ then $x \vee y=\max \{x, y\}$ and

$x \wedge y=\min \{x, y\}$ for all $x$ and $y$ in $L$. Let $(L, \leq, \vee, \wedge)$ be a lattice and $\varnothing \neq X \subseteq L$. $X$ is called a sublattice of $L$ if for any $a, b \in X, a \vee b \in X$ and $a \wedge b \in X$. A lattice $(L, \leq, \vee, \wedge)$ is said to be distributive if the operations $" \vee "$ and $" \wedge$ " are distributive with respect to each other. A matrix is called a lattice matrix if its entries belong to a distributive lattice. In this paper, the lattice $(L, \leq, \vee, \wedge)$ is always supposed to be a distributive lattice with the least and greatest elements 0 and 1, respectively. Let $M_{n}(L)$ be all $n \times n$ matrices over $L$. For any $A$ in $M_{n}(L)$, we shall denote by $a_{i j}$ or $A_{i j}$ the element of $L$ which stands in the $(i, j)$ th entry of $A$. For convenience, we shall use the set $N$ to denote the set $\{1,2, \cdots, n\}$. 
For any $A, B, C$ in $M_{n}(L)$, we define:

$A \wedge B=C$ iff $c_{i j}=a_{i j} \wedge b_{i j}$ for $i, j$ in $N$;

$A \vee B=C$ iff $c_{i j} \overline{\bar{n}} a_{i j} \vee b_{i j}$ for $i, j$ in $N$;

$A B=C$ iff $c_{i j}=\vee\left(a_{i k} \wedge b_{k j}\right)$ for $i, j$ in $N$;

$A \leq B$ iff $a_{i j} \leq b_{i j}^{k=1}$ for $i, j$ in $N$ and $A \geq B$ iff $B \leq A$

$I_{n}=\left(\eta_{i j}\right)$, where $\eta_{i j}=1$ if $i=j$ and $\eta_{i j}=0$ if $i \neq j$ for $i, j \in N$.

For any A in $M_{n}(L)$, the powers of $A$ are defined as follows: $A^{0}=I_{n}, A^{l}=A^{l-1} A, l \in Z^{+}$, where $Z^{+}$denotes the set of all positive integers. The $(i, j)$ th entry of $A^{l}$ is denoted by $a_{i j}^{l}\left(l \in Z^{+}\right)$, and

$$
a_{i j}^{l}=\underset{1 \leq i_{1}, \cdots, i_{l-1} \leq n}{\vee}\left\{a_{i_{1}} \wedge a_{i_{1} i_{2}} \wedge \cdots \wedge a_{i_{l_{-1} j}}\right\} .
$$

Let $A \in M_{n}(L), A$ is called transitive if $A^{2} \leq A$;

$A$ is called monotone increasing if $A^{2} \geq A ; A$ is called reflexive if $I_{n} \leq A$. In this paper, $A$ lattice matrix $A$ is called monotone if $A$ is transitive or $A$ is monotone increasing.

For any $A \in M_{n}(L), A$ is said to be almost periodic if there exist positive integers $k$ and $d$ such that $A^{k}=A^{k+d}$. The least positive integers $k$ and $d$ are called the index and the period of $A$, and denoted by $k(A)$ and $d(A)$, respectively. In particular, if $d(A)=1$ then $A$ is said to converges in a finite number of steps.

\section{Convergence of Monotone Lattice Matrices}

In this section, we shall discuss the convergence of Monotone Lattice Matrices. In [5,6], Tan studied the convergence index of transitive matrices and monotone increasing matrices. In the following, we continue to study the convergence index of these matrices which discussed by Tan $[5,6]$, and the convergence index of these discussed matrices is smaller than previous considered index.

Theorem 3.1. Let $A=\left(a_{i j}\right) \in M_{n}(L)$. if $a_{i i} \wedge a_{j k}=a_{j k}$ holds for all $i, j, k \in N$, then

1) $A^{2} \leq A^{3}$;

2) $a_{i i}=a_{i i}^{k}\left(\forall i \in N, k \in Z^{+}\right)$;

3) A converges to $A^{k(A)}$ with $k(A) \leq n-1$.

Proof. 1) Let

$$
a_{i j}^{2}=\underset{k=1}{\vee} a_{i k} \wedge a_{k j}(\forall i, j \in N) .
$$

By the hypothesis $a_{i i} \wedge a_{j k}=a_{j k}(\forall i, j, k \in N)$, it follows that

$$
a_{i j}^{2}=\bigvee_{k=1}^{n} a_{i k} \wedge a_{k k} \wedge a_{k j}
$$

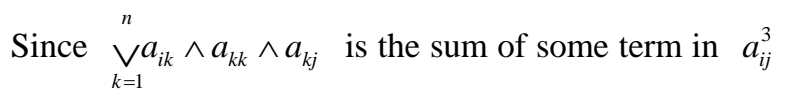
we have

$$
a_{i j}^{2}=\underset{k=1}{\vee} a_{i k} \wedge a_{k k} \wedge a_{k j} \leq a_{i j}^{3} .
$$

Thus $A^{2} \leq A^{3}$.

2) By $a_{i i} \wedge a_{j k}=a_{j k}(\forall i, j, k \in N)$, we have

$$
a_{i i} \wedge a_{i i} \wedge \cdots \wedge a_{i i}=a_{i i} .
$$

Then

$$
\begin{aligned}
& a_{i i} \leq a_{i i}^{s}\left(\forall s \in Z^{+}\right) \\
= & \underset{1 \leq i_{1}, \cdots, i_{s-1} \leq n}{\vee} a_{i i_{1}} \wedge a_{i_{1} i_{2}} \wedge \cdots \wedge a_{i_{s-1} i} \\
\leq & \underset{1 \leq i_{1} \leq n}{\vee} a_{i i_{1}}=\underset{1 \leq i_{1} \leq n}{\vee} a_{i i} \wedge a_{i i_{1}} \leq \underset{1 \leq i_{1} \leq n}{\vee} a_{i i}=a_{i i} .
\end{aligned}
$$

Therefore, $a_{i i}=a_{i i}^{s}\left(\forall s \in Z^{+}\right)$.

3) By $A^{2} \leq A^{3}$, it follows that $A^{k-1} \leq A^{K}(\forall k \geq 3)$.

Hence, $A^{n-1} \leq A^{n}$. In the following, we shall prove that $A^{n-1} \geq A^{n}$.

By the result of 2), we only need to show that $a_{i j}^{n-1} \geq a_{i j}^{n}$ for $i \neq j$. Let

$$
a_{i j}^{n}=\underset{1 \leq i_{1}, \cdots, i_{n-1} \leq n}{\vee} a_{i_{1} i_{1}} \wedge a_{i_{1} i_{2}} \wedge \cdots \wedge a_{i_{n-1} j},(\forall i, j \in N) .
$$

Since the number of indices in $a_{i i_{1}} \wedge a_{i_{1} i_{2}} \wedge \cdots \wedge a_{i_{n-1} j}$ is $n+1$, there must be two indices $i_{u}$ and $i_{v}$ such that $i_{u}=i_{v}(u<v)$. Then

$$
\begin{aligned}
& a_{i i_{1}} \wedge a_{i_{1 i_{2}}} \wedge \cdots \wedge a_{i_{n-1} j} \leq a_{i_{1}} \wedge a_{i_{1} i_{2}} \\
& \wedge \cdots \wedge a_{i_{u-1} i_{u}} \wedge a_{i_{v} i_{v+1}} \wedge \cdots \wedge a_{i_{n-1} j} .
\end{aligned}
$$

Since $a_{i i_{1}} \wedge a_{i_{1} i_{2}} \wedge \cdots \wedge a_{i_{u-1} i_{u}} \wedge a_{i_{v} i_{v+1}} \wedge \cdots \wedge a_{i_{n-1} j}$ is a term of $a_{i j}^{n-(v-u)}$, we have

$$
\begin{aligned}
& a_{i_{1}} \wedge a_{i_{1} i_{2}} \wedge \cdots \wedge a_{i_{n-1} j} \leq a_{i_{1}} \wedge a_{i_{1} i_{2}} \\
& \wedge \cdots \wedge a_{i_{u-1} i_{u}} \wedge a_{i_{v} i_{v+1}} \wedge \cdots \wedge a_{i_{n-1} j} \leq a_{i j}^{n-(v-u)} .
\end{aligned}
$$

Thus $a_{i j}^{n} \leq a_{i j}^{n-(v-u)}$, then

$$
A^{n} \leq A^{m}(m=n-(v-u)<n) \leq A^{n-1} \text { (since } A^{k-1} \leq A^{k}
$$
for $\forall k \geq 3)$.

From above, we can get $A^{n-1}=A^{n}$. This completes the proof.

Corollary 3.1. Let $A=\left(a_{i j}\right) \in M_{n}(L)$. if $A \geq I_{n}$, then

1) $A \leq A^{2}$;

2) $a_{i i}=a_{i i}^{k}=1$ for all $i \in N, k \in Z^{+}$;

3) $A$ converges to $A^{k(A)}$ with $k(A) \leq n-1$.

Proof. It follows from Theorem 3.1.

Theorem 3.2. Let $A=\left(a_{i j}\right) \in M_{n}(L)$. If $A \leq A^{2}$ and $a_{i i}=a_{i i}^{k}$ holds for all $i \in N, k \in Z^{+}$, then $A$ converges to $A^{k(A)}$ with $k(A) \leq n-1$.

Proof. Since $A \leq A^{2}$ we have $A^{i} \leq A^{i+1}\left(\forall i \in Z^{+}\right)$.

Then

$$
A^{n+1} \geq A^{n} \geq A^{m}(m \leq n-1) .
$$

Let $T=a_{i i_{1}} \wedge a_{i_{1} i_{2}} \wedge \cdots \wedge a_{i_{n-1} j}$ be any term of 
$a_{i j}^{n}(i \neq j)$.

Since the number of indices in $T$ is greater than $n$, there must be two indices $i_{u}$ and $i_{v}$ such that $i_{u}=i_{v}$ $(u<v)$. Then

$T=a_{i i_{1}} \wedge a_{i_{1} i_{2}} \wedge \cdots \wedge a_{i_{i_{u} i_{u+1}}} \wedge \cdots \wedge a_{i_{v-1} i_{v}} \wedge a_{i_{v} i_{v+1}} \wedge \cdots \wedge a_{i_{n-1} j}$. Now delete the term $a_{i_{u} i_{u+1}} \wedge \cdots \wedge a_{i_{v-1} i_{v}}$ in $T$, thus we can get a new term

$$
T^{\prime}=a_{i i_{1}} \wedge a_{i_{1} i_{2}} \wedge \cdots \wedge a_{i_{u-1} i_{u}} \wedge a_{i_{v_{v}} i_{v+1}} \wedge \cdots \wedge a_{i_{n-1} j} .
$$

Since $T^{\prime}$ is a term of $a_{i j}^{m}(m \leq n-1)$, we have $T^{\prime} \leq a_{i j}^{m}$. But by the property of the operation " $\wedge$ ", we have

$$
T \leq T^{\prime} \leq a_{i j}^{m}(m \leq n-1) .
$$

Thus $a_{i j}^{n} \leq a_{i j}^{m}$. On the other hand, by the hypothesis $A \leq A^{2}$, we have

$$
a_{i j}^{m} \leq a_{i j}^{n}(m \leq n-1) .
$$

From above, we can get $a_{i j}^{m}=a_{i j}^{n}(m \leq n-1)(i \neq j)$.

Since $a_{i i}=a_{i i}^{k}\left(\forall i \in N, k \in Z^{+}\right)$, we have

$$
a_{i j}^{m}=a_{i j}^{n}(m \leq n-1)(\forall i, j \in N),
$$

and so

$$
A^{m}=A^{n}(m \leq n-1) .
$$

This completes the proof.

Theorem 3.3. Let $A=\left(a_{i j}\right) \in M_{n}(L)$. If for any $i \in N, a_{i i}=\bigvee_{k=1}^{n}\left\{a_{i k}\right\}$ or $a_{i i}=\bigvee_{k=1}^{n}\left\{a_{k i}\right\}$, then

1) $A^{2} \leq A^{3} \leq \cdots \leq A^{n-1} \leq \cdots$;

2) $a_{i i}=a_{i i}^{k}\left(\forall i \in N, k \in Z^{+}\right)$;

3) $A$ converges to $A^{k(A)}$ with $k(A) \leq n-1$.

Proof. 1) Let

$$
a_{i j}^{2}=\bigvee_{k=1}^{n}\left\{a_{i t} \wedge a_{t j}\right\}(\forall i, j \in N) .
$$

If $a_{t t}=\bigvee_{k=1}^{n}\left\{a_{t k}\right\}$, then

$$
a_{i j}^{2}=\bigvee_{t=1}^{n}\left\{a_{i t} \wedge a_{t j}\right\}=\bigvee_{t=1}^{n}\left\{a_{i t} \wedge a_{t t} \wedge a_{t j}\right\} \leq a_{i j}^{3} .
$$

If $a_{t t}=\underset{k=1}{n}\left\{a_{k t}\right\}$, then

$$
a_{i j}^{2}=\bigvee_{t=1}^{n}\left\{a_{i t} \wedge a_{t j}\right\}=\bigvee_{t=1}^{n}\left\{a_{i t} \wedge a_{t t} \wedge a_{t j}\right\} \leq a_{i j}^{3} .
$$

Thus $a_{i j}^{2} \leq a_{i j}^{3}$, and so $A^{2} \leq A^{3}$. Therefore

$$
A^{2} \leq A^{3} \leq \cdots \leq A^{n-1} \leq \cdots .
$$

2) for any $i \in N, k \in Z^{+}$,

$$
\begin{aligned}
a_{i i}^{k+1} & =\underset{1 \leq 1_{1}, \cdots, l_{k} \leq n}{\vee}\left\{a_{i l_{1}} \wedge a_{l_{1} l_{2}} \wedge \cdots \wedge a_{l_{k} i}\right\} \\
& \leq \underset{1 \leq l_{1}, \cdots, l_{k} \leq n}{\vee}\left\{a_{i l_{1}} \wedge a_{l_{k} i}\right\} \leq\left\{\underset{1 \leq l_{1} \leq n}{\vee} a_{i l_{1}}\right\} \wedge\left\{\underset{1 \leq l_{k} \leq n}{\left.\stackrel{n}{ } a_{l_{k} i}\right\}} \leq a_{i i} .\right.
\end{aligned}
$$

On the other hand, by the result $a_{i i}^{k+1} \geq a_{i i}$ in 1 ), we have $a_{i i} \leq a_{i i}^{k+1}$.

3) It follows from Theorem 3.2. This completes the proof.

Corollary 3.2. Let $A=\left(a_{i j}\right) \in M_{n}(L)$. If for any $i, j \in N, a_{i j}=a_{i i}$ and $A \leq A^{2}$, then

1) $a_{i i}=a_{i i}^{k}(\forall k \geq 2)$;

2) A converges to $A^{k(A)}$ with $k(A) \leq n-1$.

Proof. 1) By $a_{i j}=a_{j i}(\forall i, j \in N)$, we can get

$$
a_{i i}^{2}=\bigvee_{k=1}^{n}\left\{a_{i k} \wedge a_{k i}\right\}=\bigvee_{k=1}^{n}\left\{a_{i k}\right\} \text {. }
$$

Since

$$
\begin{aligned}
& a_{i i} \leq a_{i i}^{s}(\forall s \geq 2) \\
= & \underset{1 \leq j_{1}, \cdots, j_{s-1} \leq n}{\vee}\left\{a_{i j_{1}} \wedge a_{j_{1} j_{2}} \wedge \cdots \wedge a_{j_{s-1}}\right\} \\
\leq & \underset{1 \leq j_{1}, \cdots, j_{s-1} \leq n}{\vee}\left\{a_{i j_{1}} \wedge a_{j_{s-1} i}\right\} \\
\leq & \left\{\underset{1 \leq j_{1} \leq n}{\vee} a_{i j_{1}}\right\} \wedge\left\{\underset{1 \leq j_{s-1} \leq n}{\vee} a_{j_{s-1} i}\right\} \leq\left\{\underset{1 \leq k \leq n}{\vee} a_{i k}\right\},
\end{aligned}
$$

We have $a_{i i}^{s} \leq a_{i i}^{2}(\forall s \geq 2)$. On the other hand, since $A \leq A^{2}$, we have $a_{i i}^{s} \geq a_{i i}^{2}(\forall s \geq 2)$. Therefore

$$
a_{i i}^{s}=a_{i i}^{2}(\forall s \geq 2) .
$$

2) It follows from Theorem 3.2. This completes the proof.

Theorem 3.4. If $A$ is transitive and $\operatorname{diag}(A) \leq B \leq A$. Where $\operatorname{diag}(A)=\left(c_{i j}\right)$, with $c_{i i}=a_{i i}(\forall i \in N)$ and $c_{i j}=0(i \neq j, \forall i, j \in N)$, then

1) $B$ converges to $B^{k(B)}$ with $k(B) \leq n$;

2) If $A$ satisfies $\underset{i=1}{\vee} a_{i j} \leq a_{j j}$ (or $\underset{i=1}{\vee} a_{j i} \leq a_{j j}$ ) for some $j \in N$, then $B$ converges to $B^{k(B)}$ with $k(B) \leq n-1$;

3) If $B$ satisfies $\underset{i=1}{\vee} b_{i j} \leq b_{j j}$ (or $\bigvee_{i=1}^{n} b_{j i} \leq b_{j j}$ ) for some $j \in N$, then $B$ converges to $B^{k(B)}$ with $k(B) \leq n-1$.

Proof. First by $\operatorname{diag}(A) \leq B \leq A$, we have $a_{i i}=b_{i i}(\forall i \in N)$.

1) Let

$$
b_{i j}^{n}=\underset{1 \leq i_{1}, i_{2}, \cdots, i_{n-1} \leq n}{\vee}\left\{b_{i_{i_{1}}} \wedge b_{i_{i_{1}}} \wedge \cdots \wedge b_{i_{n-1} j}\right\} .
$$

Now, we consider any term $T$ of $b_{i j}^{n}$. Since the number of indices in $T$ is greater than $n$, there must be two indices $i_{u}$ and $i_{v}$ such that $i_{u}=i_{v}(u<v)$. Then

$$
T \leq b_{i_{u} i_{u+1}} \wedge b_{i_{u+1} i_{u+2}} \wedge \cdots \wedge b_{i_{v-1} i_{u}}
$$

And

$$
T \leq b_{i_{1}} \wedge b_{i_{1} i_{2}} \wedge \cdots \wedge b_{i_{u-1} i_{u}} \wedge b_{i_{u} i_{v+1}} \wedge \cdots \wedge b_{i_{n-1} j} .
$$

Since $A$ is transitive, we have $A \geq A^{k}$ for all $k \geq 1$, and so $a_{i j} \geq a_{i j}^{k}(\forall i, j \in N)$. Thus 


$$
\begin{aligned}
& b_{i_{u} i_{u}}^{v-1} \geq b_{i_{u} i_{u}} \wedge b_{i_{u} i_{u}} \wedge \cdots \wedge b_{i_{u} i_{u}} \\
& \text { ( since } b_{i_{u} i_{u}} \wedge b_{i_{u} i_{u}} \wedge \cdots \wedge b_{i_{u} i_{u}} \text { is a term of } b_{i_{u} i_{u}}^{v-u-1} \text { ) } \\
& =b_{i_{u} i_{u}} \\
& =a_{i_{u} i_{u}}\left(\text { since } a_{i i}=b_{i i}(\forall i \in N)\right) \\
& \geq a_{i_{u} i_{u}}^{v-u} \text { (since } A \text { is transitive) } \\
& \geq b_{i_{u} i_{u}}^{v-u}(\text { since } A \geq B) \\
& \geq b_{i_{u} i_{u+1}} \wedge \cdots \wedge b_{i_{v-1} i_{u}} \\
& \geq T \text {. }
\end{aligned}
$$

Since $\quad b_{i i_{1}} \wedge b_{i_{1} i_{2}} \wedge \cdots \wedge b_{i_{u-1} i_{u}} \wedge b_{i_{u} i_{u}}^{v-u-1} \wedge b_{i_{u} i_{v+1}} \wedge \cdots \wedge b_{i_{n-1} j}$ is a term of $b_{i j}^{n-1}$, we have

$$
\begin{aligned}
& b_{i j}^{n-1} \\
& \geq b_{i i_{1}} \wedge b_{i_{1} i_{2}} \wedge \cdots \wedge b_{i_{u-1} i_{u}} \wedge b_{i_{u} i_{u}}^{v-u-1} \wedge b_{i_{u} i_{v+1}} \wedge \cdots \wedge b_{i_{n-1} j} \\
& \geq T \wedge T=T \text {. }
\end{aligned}
$$

Then $b_{i j}^{n-1} \geq b_{i j}^{n}$, and so $B^{n-1} \geq B^{n}$. Therefore $B^{n} \geq B^{n+1}$. On the other hand, since

$$
\begin{aligned}
& b_{i j}^{n+1} \geq b_{i i_{1}} \wedge b_{i_{1} i_{2}} \wedge \cdots \wedge b_{i_{u-1} i_{u}} \wedge b_{i_{u} i_{u}} \wedge b_{i_{u} i_{u+1}} \wedge \cdots \wedge b_{i_{n-1} j} \\
& \geq T \wedge b_{i_{u} i_{u}}=T\left(\text { since } b_{i_{u} i_{u}} \geq T\right) .
\end{aligned}
$$

We have $b_{i j}^{n+1} \geq b_{i j}^{n}$, then $B^{n+1} \geq B^{n}$. From above, we can get $B^{n+1}=B^{n}$, and so $k(B) \leq n$.

2) By the proof of 1 ), we have $B^{n-1} \geq B^{n}$. In the following we shall prove that $B^{n-1} \leq B^{n}$.

Let

$$
b_{i j}^{n-1}=\underset{1 \leq i_{1}, i_{2}, \cdots, i_{n-2} \leq n}{\vee}\left\{b_{i_{1}} \wedge b_{i_{1} i_{2}} \wedge \cdots \wedge b_{i_{n-2} j}\right\},
$$

Now consider any term $T=b_{i i_{1}} \wedge b_{i_{1} i_{2}} \wedge \cdots \wedge b_{i_{n-2} j}$ of $b_{i j}^{n-1}$.

a) If $i_{u}=i_{v}$ for some $u$ and $u(u<v)$, then

$b_{i_{u} i_{u}}^{v-u} \geq b_{i_{u} i_{u+1}} \wedge \cdots \wedge b_{i_{v-1} i_{u}}$

$\geq b_{i i_{1}} \wedge \cdots \wedge b_{i_{u-1} i_{u}} \wedge b_{i_{u} i_{u+1}} \wedge \cdots \wedge b_{i_{v-1} i_{u}} \wedge b_{i_{u} i_{v+1}} \wedge \cdots \wedge b_{i_{n-2} j}$

$=T$.

And so

$$
\begin{aligned}
b_{i_{u} i_{u}}= & a_{i_{u} i_{u}}\left(\text { since } a_{i i}=b_{i i}(\forall i \in N)\right) \\
& \geq a_{i_{u} i_{u}}^{v-u}(\text { since } A \text { is transitive }) \\
& \geq b_{i_{u} i_{u}}^{v-u}(\text { since } A \geq B) \geq T .
\end{aligned}
$$

$$
\begin{aligned}
& b_{i j}^{n} \geq b_{i i_{1}} \wedge b_{i_{1} i_{2}} \wedge \cdots \wedge b_{i_{u-1} i_{u}} \wedge b_{i_{u} i_{u}} \wedge b_{i_{u} i_{u+1}} \wedge \cdots \wedge b_{i_{n-2} j} \\
& \geq T \wedge b_{i_{u} i_{u}}=T .
\end{aligned}
$$

b) Suppose that $i_{u} \neq i_{v}$ for all $u \neq v$. By the hypothesis, $\bigvee_{i=1}^{n} a_{i j} \leq a_{j j} \quad\left(\right.$ or $\left.\bigvee_{i=1}^{n} a_{j i} \leq a_{j j}\right)$ for some $j \in N$ and $a_{i_{u} i_{u}}=b_{i_{u} i_{u}}$, we can get $a_{i_{u} i_{u}}=b_{i_{u} i_{u}} \geq T$, Thus

$$
\begin{aligned}
& b_{i j}^{n} \geq b_{i_{i_{1}}} \wedge b_{i_{1} i_{2}} \wedge \cdots \wedge b_{i_{u-1} i_{u}} \wedge b_{i_{u} i_{u}} \wedge b_{i_{u} i_{u+1}} \wedge \cdots \wedge b_{i_{n-2} j} \\
& \geq T \wedge b_{i_{u} i_{u}}=T .
\end{aligned}
$$

From above, we have $b_{i j}^{n} \geq b_{i j}^{n-1}$, and so $B^{n} \geq B^{n-1}$. Therefore $B^{n}=B^{n-1}$.

3) The proof of 3) is similar to that of 2). This completes the proof.

Theorem 3.4 is an improvement of Theorem 4.1 [6].

As a special of Theorem 3.4, we obtain the following Corollary.

Corollary 3.3. If $A=\left(a_{i j}\right) \in M_{n}(L)$ is transitive, then

1) A converges to $A^{k(A)}$ with $k(A) \leq n$;

2) If $A$ satisfies $\bigvee_{i=1}^{n} a_{i j} \leq a_{j j}$ (or $\bigvee_{i=1}^{n} a_{j i} \leq a_{j j}$ ) for some $j \in N$, then $A$ converges to $A^{k(A)}$ with $k(A) \leq n-1$. Corollary 3.3 is an improvement of Corollary 4.1 [6].

\section{REFERENCES}

[1] F. A. Deng and S. Y. Liu, “Application of Fuzzy Concept Networks in Fault Diagnosis," Control and Decision, Vol. 16, 2001, pp. 834-836.

[2] S. V. Ovchinnikov, "Structure of Fuzzy Binary Relations," Fuzzy Sets and Systems, Vol. 6, No. 2, 1981, pp. 169-195. doi:10.1016/0165-0114(81)90023-3

[3] V. Tahani, “A Fuzzy Model of Document Retrieval Systems," Information Processing Management, Vol. 12, No. 3, 1976, pp. 177-187. doi:10.1016/0306-4573(76)90004-2

[4] F. Harary, "On the Consistency of Precedence Matrices," Journal of the ACM, Vol. 7, No. 3, 1960, pp. 255-259. doi:10.1145/321033.321038

[5] Y. J. Tan, "On the Power of Matrices over a Distributive Lattice," Linear Algebra and Its Applications, Vol. 336, 2001, pp. 1-14. doi:10.1016/j.laa.2004.11.016

[6] Y. J. Tan, "On the Transitive Matrices over Distributive Lattices,” Linear Algebra and Its Applications, Vol. 400, 2005, pp. 169-191.

Then 